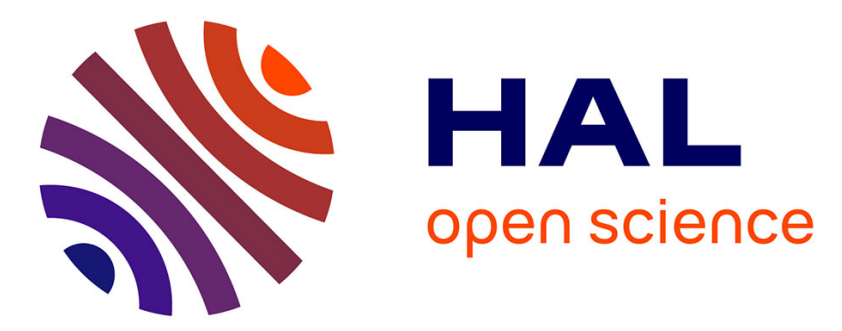

\title{
Advances in treatment formulations for acute myeloid leukemia
}

Thomas Briot, Emilie Roger, Sylvain Thépot, Frédéric Lagarce

\section{To cite this version:}

Thomas Briot, Emilie Roger, Sylvain Thépot, Frédéric Lagarce. Advances in treatment formulations for acute myeloid leukemia. Drug Discovery Today Biosilico, 2018, Epub ahead of print. 10.1016/j.drudis.2018.05.040 . inserm-01809889

\section{HAL Id: inserm-01809889 https://www.hal.inserm.fr/inserm-01809889}

Submitted on 7 Jun 2018

HAL is a multi-disciplinary open access archive for the deposit and dissemination of scientific research documents, whether they are published or not. The documents may come from teaching and research institutions in France or abroad, or from public or private research centers.
L'archive ouverte pluridisciplinaire HAL, est destinée au dépôt et à la diffusion de documents scientifiques de niveau recherche, publiés ou non, émanant des établissements d'enseignement et de recherche français ou étrangers, des laboratoires publics ou privés. 


\section{Accepted Manuscript}

Title: Advances in treatment formulations for acute myeloid leukemia

Authors: Thomas Briot, Emilie Roger, Sylvain Thépot, Frederic Lagarce

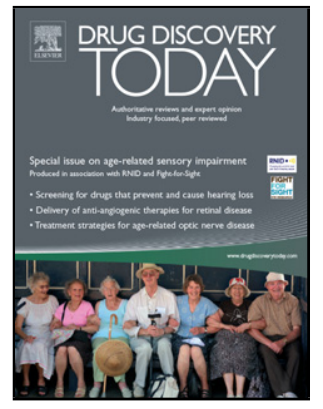

PII: S1359-6446(18)30085-0

DOI: https://doi.org/10.1016/j.drudis.2018.05.040

Reference: DRUDIS 2262

To appear in:

Please cite this article as: Briot, Thomas, Roger, Emilie, Thépot, Sylvain, Lagarce, Frederic, Advances in treatment formulations for acute myeloid leukemia.Drug Discovery Today https://doi.org/10.1016/j.drudis.2018.05.040

This is a PDF file of an unedited manuscript that has been accepted for publication. As a service to our customers we are providing this early version of the manuscript. The manuscript will undergo copyediting, typesetting, and review of the resulting proof before it is published in its final form. Please note that during the production process errors may be discovered which could affect the content, and all legal disclaimers that apply to the journal pertain. 


\title{
Advances leukemia
}

\author{
Thomas Briot ${ }^{1,2}$, Emilie Roger ${ }^{1}$, Sylvain Thépot ${ }^{3,4}$, and Frederic Lagarce ${ }^{1,2}$
}

\author{
${ }^{1}$ Micro \& Nanomédecines Translationelles-MINT, UNIV Angers, INSERM 1066, CNRS 6021, Université Bretagne \\ Loire, MINT IBS-CHU, 4 rue Larrey, 49933 Angers, France \\ ${ }^{2}$ University Hospital of Angers, Pharmacy department, 4 rue Larrey, 49933 Angers, France \\ ${ }^{3}$ University Hospital of Angers, clinical hematology department, 4 rue Larrey, 49933 Angers, France \\ ${ }^{4}$ INSERM CRCINA, University of Angers, 4 rue Larrey, 49933 Angers, France
}

\section{Corresponding author: Lagarce, F. (frederic.lagarce@univ-angers.fr)}

Acute myeloid leukemia (AML) is the most common cause of leukemia-related mortality. The combination of cytarabine and anthracycline has been the gold standard of treatment over the past $\mathbf{4 0}$ years, but the distribution of the drugs in the body leads to severe adverse effects. Poor prognosis of older patients with AML is the consequence not only of comorbidities, but also of chemoresistance resulting from frequent secondary AML. Numerous strategies using nanotechnologies are in development to improve drug targeting, pharmacokinetics, administration route, chemoresistance, and adverse effects generally observed. Among the four new drugs approved for AML by the US Food and Drug Administration (FDA) in 2017, Vyxeos $^{\circledR}$ is a novel liposomal formulation of historical AML drugs. Here, we review current AML treatments and discuss how the development of new formulations will change the therapeutic armamentarium.

Keywords: acute myeloid leukemia; formulations; liposomes; nanoparticles; micelles; nanocapsules.

Teaser: This review presents current treatments generally used in acute myeloid leukemia therapy, and highlights innovative formulations designed to overcome drug resistance or adverse effects observed with current treatments.

\section{Introduction}

AML is the most common acute leukemia in adults, and its incidence increases with age. Although the disease is usually of unknown etiology, it can develop following either an underlying hematological disorder (e.g., myelodysplastic syndromes, MDS) or exposure to genotoxic agents (e.g., topoisomerases II, alkylating agents, or radiation). The pathogenesis of AML involves successive genomic alterations in multipotential hematopoietic stem cells and leads to the abnormal clonal proliferation and differentiation of myeloid cells.

AML mainly affects adult patients, with a median age of 64 years [1]. Prognosis remains poor, especially in older patients, with fewer than $30 \%$ of patients surviving within a year of diagnosis [2].

Although our understanding of the disease as well as its biological characteristics has advanced in recent decades, induction therapy has changed little since 1973. However, the therapeutic landscape of AML dramatically changed in 2017 with four novel drugs approved by the FDA. Among them, Vyxeos® (Jazz Pharmaceuticals) is a novel liposomal formulation of a co-encapsulation of daunorubicin and cytarabine.

The principal adverse effect generally observed in AML treatment is myelosuppression with neutropenia, which can lead to severe infections, anemias requiring red blood cell transfusions, and thrombocytopenia involving hemorrhagic events. Supportive care with anti-infectious treatments, such as antifungals, antibiotics, and antivirals $[3,4]$ and growth factors [5] has led to improved overall survival rates for patients with AML over recent decades $[6,7]$.

Here, we highlight innovative formulations as a potential way to optimize AML chemotherapies. Specifically, we focus on how nanoparticles (NPs) might be designed to counteract adverse effects, enhance treatment compliance, avoid resistance, or prolong exposure to chemotherapy.

\section{Current treatments}

In younger patients, AML requires the instauration of therapy with a minimal delay after diagnosis [8]. By contrast, delaying AML treatment does not affect prognosis in older patients, except those with very proliferative disease [9]. Intensive chemotherapy is the gold standard for younger patients with AML, whereas epigenetic therapy is an option for older patients, especially those with comorbidities or belonging to high-risk populations (i.e., secondary AML or poor risk cytogenetics) [10]. 


\section{Standard chemotherapy}

Induction therapy for younger patients with AML in 2018 remains largely as it was during the 1970s [11]. The most common therapy used in AML is the ' $7+3$ regimen', a combination of cytarabine (a nucleoside analog) combined with daunorubicin or idarubicin (an anthracycline) [12]. Patients eligible for intensive chemotherapy usually undergo this treatment, which comprises a continuous infusion of cytarabine $\left(100-200 \mathrm{mg} / \mathrm{m}^{2}\right)$ for 7 consecutive days and daunorubicin at $60 \mathrm{mg} / \mathrm{m}^{2}$ or idarubicin at $12 \mathrm{mg} / \mathrm{m}^{2}$ on Days $1-3$. Reducing daunorubicin to $45 \mathrm{mg} / \mathrm{m}^{2} \mathrm{per}$ day is considered suboptimal [13], whereas it is thought that idarubicin is associated with a higher cure rate in the long term [14]. After induction, complete remission is achieved in 60-80\% of younger patients with AML, compared with approximately $45-65 \%$ in older patients $[13,15,16]$. This induction is then followed by consolidation therapy during the postremission phase. This therapy comprises two to four cycles of high-dose cytarabine $\left(2000-3000 \mathrm{mg} / \mathrm{m}^{2} / \mathrm{day}\right.$ given on Days 1, 3, and 5) and can be followed by hematopoietic stem cell transplantation depending on age, AML risk group, and donor availability.

\section{Specific therapy}

The introduction of all-trans retinoic acid (ATRA) into the therapy of acute promyelocytic leukemia (APL), which represents $10-15 \%$ of all cases of AML, completely revolutionized the management and outcomes of this disease [17]. ATRA combined with anthracyclines results in a remission rate of $>90 \%$, and provides the first example of molecularly targeted treatment in AML [18]. ATRA, administered orally at $45 \mathrm{mg} / \mathrm{m}^{2}$ twice daily, is presented as 10 mg soft capsules in which ATRA is solubilized in a mixture of yellow beeswax, hydrogenated soya bean oil, partially hydrogenated soya bean oil, and soya bean oil. ATRA has also been combined with arsenic trioxide (ATO), as a result of synergy in inducing differentiation and apoptosis. The combination of ATRA with ATO resulted in the first chemotherapy-free regimen for AML and has become the new standard for APL [19] with inferior toxicity compared with ATRA combined with chemotherapy [20,21].

Relevant molecular targets have been discovered in AML. For example, isocitrate dehydrogenases (IDHs) can be mutated, leading to the accumulation of R-2-hydroxyglutarate in the cell. Mutations of two isoforms of IDHs (IDH1 and IDH2) have been linked to epigenetic changes resulting in global dysregulation of gene expression. Those mutations have been found in approximately $20 \%$ of patients with newly diagnosed AML [22]. FMS-like tyrosine kinase 3 (FLT3) is a gene that encodes a tyrosine-kinase and is mutated in some patients with AML. Mutant forms of FLT3 can be targeted by kinase inhibitors [23].

Midostaurin was the first kinase inhibitor approved for AML by the FDA and the European Medicines Agency (EMA), in 2017. This drug is given orally at $50 \mathrm{mg}$ twice daily in association with the conventional ' $7+3 \mathrm{regimen',} \mathrm{in}$ AML with FLT3 internal tandem duplication or tyrosine kinase activation. In the RATIFY trial, the median overall survival was prolonged from 25.6 to 74.7 months with midostaurin in patients aged 18-59 years [24].

The FDA also provided accelerated approval for the oral IDH2 inhibitor enasidenib in August 2017, based on a promising Phase 2 study in relapsed or refractory AML [25]. Given orally at $100 \mathrm{mg}$ per day, alone, this treatment produced an overall response rate of $37 \%$. Based on these results, this treatment is likely to be combined in the near future with conventional chemotherapy or demethylating agents in patients with first-line AML and IDH2 mutations.

Other targeted agents inducing mutations are under development, such as IDH1 inhibitors (ivosidenib) [26] and BCL2 antagonism (venetoclax) either alone or in combination [27-29]. Interesting reviews of new active pharmaceutical ingredients (API) used in AML treatment are available elsewhere [30,31].

\section{Antibody-drug conjugates}

Gemtuzumab ozogamicin (GO) was the first antibody-drug conjugate approved by the FDA for CD33-positive AML. CD33 is a membrane receptor expressed by myeloid progenitors but not present in normal hematopoietic stem cells. This drug is a humanized anti-CD33 antibody conjugated to calicheamicin (a DNA-intercalating antibiotic). Accelerated approval was given by FDA in 2000 but withdrawn 10 years later because of increased treatment-related mortality [32]. Final approval was given in 2017 based on new GO dose regimens [33]. In addition to new CD33targeting agents, other clinical trials targeting CD123 are ongoing [34].

\section{Epigenetic therapy}

For patients who are not candidates for intensive chemotherapy, such as older patients with comorbidities or relapsed or refractory AML, lower intensity therapy is required. For several years, the only solutions available were bestsupportive care or low-dose cytarabine. Lower-intensity AML treatment is currently represented by hypomethylating agents, such as decitabine (DEC) and azacytidine (AZA). The role of epigenetic deregulation in the pathogenesis of AML and MDS has been a focus of intense research over the past 10 years. Initially developed for patients with higher-risk MDS, DEC and AZA have shown their ability to improve median overall survival compared with lowdose cytarabine or supportive care only [35,36]. In AML, both hypomethylating agents have been compared with conventional care regimens in two Phase 3 trials [10,37], in which they displayed an improvement in median overall survival rates as well as lower toxicity. Whereas DEC injected intravenously at $20 \mathrm{mg} / \mathrm{m}^{2}$ every day over 5 days, repeated every 4 weeks, was approved in 2012 by the EMA for older patients with de novo AML who were not candidates for standard induction therapy [38], it is only approved by the FDA for patients with MDS. AZA (75 mg/m²) subcutaneously for 7 days every 28 days is also approved in the USA and Europe for MDS, but not for AML. However, patients with $20-30 \%$ bone marrow blasts (refractory anemia with excess blast in transformation according to 
French-American-British subtypes) are now considered patients with AML according to the WHO's classification [39], meaning that the use of DEC and AZA is a labeled option.

Other novel agents currently under investigation in clinical trials were recently reviewed by Montalban et al. [40].

\section{Questions and challenges for AML treatment}

Chemotherapies used in AML are mostly presented as solutions and are administered intravenously. A general distribution is mostly observed without drug targeting towards specific cells. Therefore, these chemotherapies can lead to general adverse effects, such as pancytopenia or cardiac toxicities related to anthracyclines. Treatmentrelated mortality is high, especially in the older population. However, specific AML therapies are mostly oral treatments, although the new schedules developed for patients with AML are also affected by adverse effects and drug resistance. Tyrosine kinase inhibitors are associated with digestive adverse effects (vomiting, nausea, diarrhea, and heartburn), various types of skin rash, headaches, muscle cramps, heart dysfunction, hepatic impairments, pulmonary toxicities, or cardiotoxicities [40-42].

Despite new treatments, personalized therapies and many clinical trials, overall survival remains poor. Complete remission is reached in 70-80\% of younger patients with AML ( $<60$ years), compared with only 45-65\% of older patients with AML because of comorbidities and drug resistance [13,43]. Moreover, according to European Leukemia Net, median overall survival falls from 15.5 to 5.5 years in favorable AML genetic groups among patients over the age of 60 [44]. Relapse occurs when drugs are unable to eradicate leukemic stem cells (LSCs) protected in the hematopoietic niche. One of the major causes of drug resistance is the altered function of transmembrane transporters. Several members of the ATP-binding cassette transporters family are upregulated in tumor cells [45], and could be a target in AML [46].

To bypass these phenomena, pharmaceutical technologies, such as nanotechnologies, are being developed, and this field represents a promising approach [47]. In particular, in AML therapy, nanocarriers could prolong the half-life of drugs generally administered via an intravenous administration, thus enhancing the contact of the drug with cells. Specific cells, such as LSCs, could be targeted to reduce relapses [48,49]. Finally, modifying the administration route can help to improve patient compliance, especially of older patients.

\section{Challenges for innovative formulations}

According to the definition given by the FDA and the EMA, nanomaterials involved in drug delivery should have at least one external, internal, or surface structure in the nanoscale range (1-100 nm). Nanomaterials involved in drug development can include liposomes, lipid NPs, polymeric NPs, micelles, dendrimers, and metallic NPs, depending on their formulation process and their composition. Since the first nanocarriers were developed, several approaches have emerged to enhance their effectiveness, depending on the purpose of the nanocarrier. Typically, four generations of NPs have been distinguished over time. In the first one, particles were 'nude' and rapid opsonizations were observed. In the second generation, hydrophilic polymers were grafted onto the surfaces of NPs to form a brush-like barrier. Slowed protein adsorption onto NPs was achieved, and mean blood residence times were enhanced. Secondgeneration particles diffuse to solid tumors because of the enhanced permeability and retention (EPR) effect. However, this effect does not appear to work in human therapeutics [50] and has no implications for therapeutics in AML, a nonsolid tumor cancer. Third-generation particles comprise NPs with specific ligands on their surfaces, designed specifically to target cells of interest. The fourth generation corresponds to theranostic strategies (multifunctional nanocarriers that combine a diagnostic agent with a therapeutic agent) or targeting cell organelles [51].

Several nanocarrier formulations have been approved and are available on the US, European, Indian, Japanese, or Chinese markets. They are mostly liposomal formulations and are largely used for solid tumors. Nanocarriers are generally designed for four main reasons: (i) drug targeting with the aim of reducing adverse effects resulting from the general distribution of the API or from excipients; modify drug pharmacokinetics; (3) bypass drug resistance; and (4) modify the route by which the drug is administered or to improve the oral bioavailability of APIs [52]. This can lead to improved quality of life for patient if intravenous administration is replaced by oral administration.

Other hematologic malignancies have also been treated using liposomal formulations. For example, liposomal pegylated doxorubicin formulations, indicated in multiple myeloma, decrease the cardiac toxicity associated with anthracycline. However, new adverse effects have been observed with these formulations, such as acute hypersensitivity reactions. Liposomal formulations modify doxorubicin pharmacokinetics by increasing its half-life and plasma concentration (area under curve increased) and decreasing the volume of distribution [53]. Moreover, doxorubicin-loaded liposomes are less subject than free doxorubicin to multidrug resistance mechanisms driven by p-glycoprotein, and are capable of reversing multidrug resistance efflux mechanisms [54].

There are a few NP formulations on the market, and one, Vyxeos ${ }^{\circledR}$, was approved for AML treatment by the FDA in 2017. This liposomal formulation is a co-encapsulation of cytarabine and daunorubicin, and is discussed in more detail below.

Forty-two clinical trials corresponding to a combination of the search terms 'acute myeloid leukemia' and 'liposomes', 'nanoparticles', 'nanocarriers', 'micelles' or 'nanocapsules' were found on the clinicaltrials.gov website in December 2017 (Table 1). Among the 42 clinical trials, eight corresponded to antifungal drugs, and six did not used nanocarrier formulations but were found because of their use of associated keywords (MESH terms). Finally, 28 clinical trials were identified that used liposomal formulations, but only one was based on polymeric NPs. No other NP formulation is currently under clinical investigation for the treatment of AML. 


\section{Liposomes}

Liposomes are vesicles generally comprising phospholipids and cholesterol in a bilayer arrangement (Figure 1). Liposomes can be loaded with one or more molecules in the aqueous compartment or in the lipidic bilayer. Liposomal formulations are already used in clinical settings for non-AML indications, such as anti-infectious drug encapsulation (amphotericin B, Ambisome ${ }^{\circledR}$, Astellas Pharma), analgesic drugs [morphine sulfate, Depodur ${ }^{\circledR}$, Pacira Pharmaceuticals (commercialization discontinued)] or cytotoxic drugs. In terms of cytotoxic drugs, only two drug classes have been encapsulated into liposomes; anthracycline and antimetabolite agents. Two anthracycline molecules were encapsulated and reached the market, namely doxorubicin (Caelyx ${ }^{\circledR}$, Janssen-Cilag International N.V, Doxil ${ }^{\circledR}$, Janssen-Cilag International N.V, and Myocet ${ }^{\circledR}$, Teva B.V.) and daunorubicin (Daunoxome ${ }^{\circledR}$, Gilead Sciences) for which commercialization was discontinued. Cytarabine (DepoCyt囚, Pacira Ltd) is the only representative drug of the antimetabolite class encapsulated in a liposome-like structure (nonconcentric vesicles). The commercialization of Depocyt ${ }^{\circledR}$ was discontinued in 2017. The main advantages of liposomes are that they have shown a good ability for large-scale production (i.e., the presence of drug products on the market) and also their tolerance is now well studied.

Liposomes approved as AML therapy In 2017, the FDA approved Vyxeos ${ }^{\circledR}$ (previously denoted CPX-351), a liposomal formulation with a co-encapsulation of cytarabine and daunorubicin in a 5:1 molar ratio. To obtain theses liposomes, an emulsion was formed comprising distearoylphosphatidylglycerol, distearoylphosphatidylcholine, and cholesterol, solubilized in chloroform and dispersed in a solution comprising copper gluconate and triethanolamine. The solvent (chloroform) is evaporated and the film is then rehydrated and the liposomes extruded on a polycarbonate filter [55]. Vyxeos ${ }^{\circledR}$ is presented in a lyophilized form and should be reconstituted with water for injection; it should then be diluted in $0.9 \%$ sodium chloride injection or $5 \%$ dextrose injection before intravenous injection. The mean size of liposomes is $107 \mathrm{~nm}$, with a polydispersity index (PI) of 0.243 and a negative surface charge (potential zeta of -33 $\mathrm{mV}$ ) [55]. In a Phase -2 randomized trial, CPX-351 was compared with a ' $7+3$ regimen' in older patients with firstline AML [56]. This clinical trial enrolled 127 patients, of whom 86 were allocated to the CPX-351 arm and 41 to the gold standard treatment arm. Two courses of induction and consolidation were allowed in each arm. CPX-351 was associated with a higher complete remission rate and a lower 30-day treatment-related mortality rate (3.5\% versus 7.3\%). It was demonstrated that liposomes can enter leukemic cells intact, and this mechanism was suggested as a means of overcoming drug resistance [57]. Indeed, anthracyclines are susceptible to efflux pump (because of the pglycoprotein) and, once encapsulated into liposomes, cellular internalization is mediated by liposome properties rather than by anthracycline properties. Thus, daunorubicin was able to escape to the p-glycoprotein. Cytarabine is rapidly metabolized by cytidine deaminase and its encapsulation into liposomes can protect it from this degradation pathway. CPX-351 was also evaluated in first relapse in younger patients with AML aged 18 to 65 [58]. This Phase 2 trial was based on 2:1 randomization [i.e., CPX-351 or an alternative chosen by the investigator and based mainly on cytarabine (97.3\%) and anthracycline (77.3\%)]. As in the previous clinical trial, a higher number of complete remissions was observed in the CPX-351 arm, along with a lower number of deaths after 90 days. A Phase 3 clinical trial was then conducted in patients aged 60 to 75 with untreated high-risk or secondary AML (NCT 01696084). This trial recruited 309 patients and confirmed the higher complete remission rate (47.7\% versus $33.3 \%)$ and overall survival (median, 9.56 versus 5.95 months) for CPX-351 over the ' $7+3$ regimen' $[31,59,60]$.

Vyxeos ${ }^{\circledR}$ is now labeled for the treatment of adults with newly diagnosed therapy-related AML or AML with myelodysplasia-related changes. According to cliniclatrials.gov, in December 2017, 11 clinical trials were ongoing using the liposomal formulation of daunorubicin and cytarabine (Table 1), with the aim of expanding Vyxeos $\mathbb{R}$ indications.

Liposomes under clinical trial as AML therapy Other liposomal formulations are under clinical evaluation for AML indications (Table 1). These are drug formulations already on the market and are used in clinical trials as drug support to investigate other new agents, to develop new schedule strategies, or to investigate new chemotherapeutic agent associations. Vincristine-sulfate liposome, annamycin liposomes, or BP1001 (a liposomal growth factor receptor-bound protein-2 antisense oligodeoxynucleotide loaded in a liposome formulation) are new drugs that are not yet approved. They are currently at the Phase 1 and 2 evaluation stages to determine either the maximum tolerated dose (annamycin liposomes and BP1001) or their efficacy (vincristine sulfate liposomes) in patients with relapsed or refractory AML.

Liposomes can be used for co-encapsulation, and Vyxeos ${ }^{\circledR}$ is proof that co-delivery could be more effective than a combination of drugs administered separately, probably because the molar ratio is preserved throughout drug release [61]. Moreover, Vyxeos ${ }^{\circledR}$ confirms that using nanotechnologies could overcome drug resistance, such as p-glycoprotein (anthracycline), and protect drugs from enzymatic degradation pathways, such as cytidine deaminase (cytarabine).

Liposomes evaluated in in vitro or in vivo models Other liposome formulations have been developed for AML treatment, and are designed to avoid adverse effects. For example, a method has been developed for the co-delivery of safingol and C2-ceramide [62]. Safingol is a sphingolipid with antileukemic activity, but is not used in the clinical setting because of its poor water solubility and hemolytic properties [63]. Tan et al. designed liposomes for safingol encapsulation. These were prepared by mixing safingol, distearoylphosphatidylcholine, and cholesterol in chloroform before evaporating the latter. The lipid film was then rehydrated in a HEPES buffer, which was followed by extrusion techniques on polycarbonate filters. Finally, liposomes with a positive zeta potential were obtained. The liposomes 
were able to reduce significantly the in vitro hemolytic toxicity of safingol and safingol plasma elimination in a mousebased model, and to improve survival in U937-inoculated mice (histiocytic lymphoma cells) [63]. The same authors then developed a co-encapsulation of safingol and C2-ceramide (1:1 molar ratio), another sphingolipid [62]. Given that C2-ceramide and safingol act independently, with different cellular pathways, the authors postulated that coencapsulation of these two sphingolipids would enable them to act in synergistically. A liposomal formulation was obtained after the dissolution of the lipids (safingol, C2-ceramide, cholesterol, and distearoylphosphatidylcholine) in chloroform. The solvent was then evaporated, and the film obtained was rehydrated with piperazineethanesulfonic acid buffer. The liposomes were then extruded on polycarbonate filters. Liposomes of $135 \mathrm{~nm}$ with a co-encapsulation of safingol and C2-ceramide were obtained that were similar to liposomes containing only C2-ceramide (119 nm). Liposomes significantly reduced the hemolysis properties of sphingolipids compared with free drugs. The concentrations required for liposomal $\mathrm{C} 2$-ceramide to achieve 50\% cell death were significantly higher than those for liposomal co-encapsulation of safingol and C2-ceramide on five leukemia cell lines (KG-1, MV-4-11, HL-60, U937, and HEL). Finally, in vivo experiments were carried out involving intravenous inoculation of U937 using a mousebased model. Despite a reduction in the drug doses administered, co-encapsulation extended the median survival time from 24 to 37 days compared with liposomes with $\mathrm{C} 2$-ceramide encapsulation alone $(P<0.005)$, demonstrating once again the potential of co-encapsulation into liposomes.

Myhren et al. also developed a liposome formulation for the co-administration of an anthracycline (daunorubicin) and a protein synthesis inhibitor, emetine [64]. Liposomes were grafted onto the liposome surface with polyethylene glycol (PEG) to confer stealth properties, and folic acid was also grafted on to target AML cells. The liposomes comprised phosphatidylcholine, cholesterol, and distearoyl phosphatidylethanolamine or distearoyl-glycerophosphoethanolamine-N-PEG5000-folate. After the lipids were solubilized in chloroform, the solvent was removed, and the film obtained was rehydrated in ammonium sulfate buffer and then extruded. Liposomes were then loaded with drugs by applying the acid-precipitation concept. Finally, liposomes with a size of 121-127 nm (depending on the folic acid grafting), and a negative charge of approximately $-10 \mathrm{mV}$ were obtained. After an in vitro study on an AML cell line (MOLM-13), the authors showed that liposomes with a co-encapsulation were more effective than a coincubation of daunorubicin-loaded liposomes and emetine-loaded liposomes. Apoptosis was enhanced by a factor of six. However, the authors also showed that incubation of daunorubicin-loaded liposomes followed 30 min later by incubation of emetine-loaded liposomes was more effective than the administration of liposomes containing the two drugs.

Liposomes and biopharmaceuticals Biopharmaceuticals are currently a research focus and liposomes could help in administering these APIs. Biotechnology is an emerging field with great potential in cancer treatment, and AML is affected by this development. Biotechnology encompasses several treatment strategies, such as gene therapy, antisense oligonucleotide therapy, and chimeric antigen receptor $\mathrm{T}$ cells, which were recently approved for acute lymphoblastic leukemia treatment by the FDA. Antisense oligonucleotides refer to small interfering (si)RNA, miRNA, or short hairpin (sh)RNA. There are few antisense oligonucleotides on the market, probably because of a lack of stability in blood circulation, inefficient intracellular delivery, difficulties in targeting cells or tissues, and poor affinity with the target sequence [65]. Therefore, as part of cancer treatment strategies, different NPs were designed to improve either the stability in the blood stream or their ability to target cancer cells [66]. Hundreds of Phase 1, 2, and 3 clinical trials for cancer indications are ongoing [67]. However, only two clinical trials combining liposomes and antisense oligonucleotide for AML treatment were found on clinicaltrials.gov in December 2017 (Table 1).

BP1001 is a liposomal formulation of an antisense oligonucleotide (growth factor receptor-bound protein-2) comprising dioleoylphosphatidylcholine, with a mean size $<900 \mathrm{~nm}$, and high encapsulation efficiency (>95\%) [68]. Two clinical trials are ongoing. A Phase 1 trial is underway (NCT01159028) to determine the highest safe dose for patients with Philadelphia chromosome-positive chronic myeloid leukemia, AML, acute lymphoblastic leukemia, and MDS (active but not recruiting patients). The Phase 2 trial (NCT02781883) is designed for patients with newly diagnosed AML who are not suitable for standard or high-intensity chemotherapy regimens (currently recruiting patients). The study is designed to assess whether the combination of BP1001 and low-dose cytarabine is more effective than low-dose cytarabine alone.

Other formulations are under in vitro or in vivo evaluation. miR-126 is overexpressed in LSC [69] and the cells appear to be engaged in AML treatment resistance; thus, targeting LSCs appears to be a promising therapeutic approach [70]. Dorrance et al. designed lipoplex targeting LSCs, and more specifically miR-126, with the objective to reduce quiescent LSC and leukemia growth [49]. A negatively charged miR-126-antagonist was mixed with positively charged polyethylamine, producing a polyplex core that was then added to an anionic liposomal formulation, finally resulting in a lipopolyplex. To target LSC, transferrin or CD45 antibody was then grafted onto the surface of the lipoplex. The formulations were tested on mice engrafted with human AML primary blasts. The mice were treated with lipopolyplex loaded with anti-miR-126 or with anti-miR-scramble (SCR) as a control. After 48 h of treatment, bone marrow cells were collected and transplanted into secondary mice. Whereas a significant improvement in survival rate of secondary recipients was observed, there was no impact for primary recipient mice transplanted with cells harvested after an anti-miR-126-lipopolyplex formulation compared with mice transplanted with cells harvested after an anti-miR-SCR-lipopolyplex formulation. Thus, this combination of anti-miR-126 and chemotherapy could be an effective way to target LSCs and decrease leukemia relapses.

Liposomes were also developed to encapsulate GTI-2040 (also known as LOR-2040) and to target ribonucleotide reductase, which is overexpressed in cytarabine-resistant AML cells [71]. GTI-2040, targeting the R2 ribonucleotide 
reductase subunit, was evaluated in a Phase 1 clinical trial in combination with cytarabine. R2 downregulation was observed but no complete remission was noted, indicating that the effect of GTI-2040 needed to be enhanced [72]. GTI-2040-loaded immunoliposomes, grafted with an anti-CD33 to target AML cells, were designed and evaluated by $\mathrm{Li}$ et al. [71]. These liposomes were previously formulated using an emulsion comprising lipids (2,3-dioleyloxy-propyltrimethylammonium chloride, dioleoylphosphatidylethanolamine, $\alpha$-tocopheryl PEG-1000 succinate, and cholesterol) solubilized in ethanol and then injected in an HEPES buffer. Mean particle size was $93 \pm 18 \mathrm{~nm}$, and the zeta potential was relatively neutral $(4.3 \pm 0.2 \mathrm{mV})$. After cytotoxicity testing on Kasumi-1 (an AML cell line) and K562 (chronic myelogenous leukemia cell line), the authors developed an in vivo model of a Kasumi-1 xenografted tumor. Following daily administration of GTI-2040-loaded immunoliposomes for 3 days, significantly downregulated R2 expression was observed compared with free GTI-2040. Tumor volume decreased by a factor of approximately two. When combined with cytarabine treatment, the survival of mice was also prolonged with the use of GTI-2040-loaded immunoliposomes compared with cytarabine alone, cytarabine plus free GTI-2040, or cytarabine plus GTI-2040loaded liposomes without anti-CD33 grafting.

Finally, several liposomal formulations using chemotherapeutic drugs or antisense oligonucleotides are in various stages of development (Table 2). Combination therapy with drug co-encapsulation or drug co-administration is now offered on a systematic basis. Liposomes have been designed to enhance drug targeting and to overcome drug resistance, and their development is continuing, with several formulations currently under clinical evaluation.

\section{Nanoparticles based on polymers}

Those particles in the nano-size range contain supramolecular associations of polymers (micelles and dendrimers) and aggregated dense polymeric particles. The stability of these formulations in the body can be very different, as can their toxicity and scale-up ability. Thus, the future of these formulations in the clinic is linked not only to their performance, but also to their fate in the human body, resulting from their physicochemical properties. The difficulties in producing and accurately characterizing large-scale batches could hinder their future development.

Polymeric NPs Polymeric NPs (Figure 1) are NPs formulated from polymers and designed to encapsulate hydrophilic or lipophilic drugs depending on the formulation process used. Along with liposomes, polymeric NPs represent the most significant nanomedicine categories approved by the FDA [73].

For AML indications, one clinical trial using polymeric NPs has been registered (Table 1), and others are currently being investigated in preclinical models.

Polymeric NPs encapsulating AZD2811, a molecularly targeted agent (aurora B kinase inhibitor), have been developed and will be evaluated in an approved clinical trial (NCT03217838). NPs comprise block copolymers of polyD,L-lactic acid (PLA) and PEG. A nanoemulsion of benzyl alcohol and an aqueous phase containing an organic acid have previously been obtained. The organic acid led to a counterion interaction with AZD2811, increasing drug encapsulation efficiency and decreasing the release rate of the drug [74]. An in vivo pharmacokinetic study using a rat-based model confirmed the slow release of the drug, extending its exposure profile. Pharmacodynamic studies in multiple preclinical models have demonstrated that AZD2811-loaded NPs were more effective in inhibiting tumor growth when administered at half of the dose of AZD1152. AZD1152 corresponds to the AZD2811 phosphorylated prodrug (also known as barasertib) [74]. In a HL-60-xenografted mouse-based model, AZD2811 NPs showed the potential to improve AZD2811 efficacy in regressing tumor size at the various doses investigated, as well as being well tolerated [75]. When combined with cytarabine in the same model, tumor size regression and the sustainability of the response were increased compared with cytarabine alone or AZD2811 NPs alone. When AZD1152 (used at a higher total dose compared with AZD2811 NPs) was combined with cytarabine, the duration of the response was reduced, confirming the potential of AZD2811-PLA NPs [75].

Simon et al. developed NPs to encapsulate ATRA in polymeric NPs based on poly-DL-lactide-co-glycolide (PLGA) polymer [76]. PLGA NPs were obtained using the solvent evaporation technique. After a lyophilization process, the mean particle size was 150-200 nm. The NPs did not affect the antiproliferative and differentiation capabilities of ATRA in HL-60 cells when entrapped. Given that PLGA NPs are already used for parenteral administration, and PLGA is a FDA-approved polymer for parenteral use, the authors concluded that ATRA polymeric NPs could be useful for intravenous administration, particularly because the release of the drug was prolonged. Currently, ATRA is only available for oral administration, and a parenteral administration method could be required for patients who are unable to swallow.

Multifunctional lipid-polymeric NPs loaded with doxorubicin and obtained by the nanoprecipitation method have also been developed [77]. In this strategy, doxorubicin was encapsulated in a polymeric core comprising PLGA and Pluronic ${ }^{\circledR} 85$ (co-polymers based on ethylene oxide and propylene oxide). Transferrin was then grafted onto a lipidic shell comprising stearic acid, after being conjugated to PEG and oxalic acid. The grafting led to the targeting of transferrin receptors expressed in leukemic cells. Particles had a mean size of $160 \mathrm{~nm}$ and a negative charge. The formulation showed the ability to prolong doxorubicin plasma circulation time in a rat-based model. The mean residence time was enhanced by a factor of at least five. Tumor reduction volume in a mouse-based model xenografted with HL-60 cells or doxorubicin-resistant HL-60 cells was also enhanced. Doxorubicin multidrug resistance was also overcome, probably because of the presence of Pluronic ${ }^{8} 85$ in the core of the NPs, which is able to inhibit pglycoprotein.

A specific CD44 siRNA to target LSCs loaded in polymeric NPs has also been developed [78]. CD44, an adhesion molecule, is associated with poor AML prognosis and high level of AML relapse [79]. Lipid-modified 
polyethyleneimine dissolved in water was added to CD44-specific siRNA, solubilized in a saline medium [80]. The mean size of the complexes obtained was $<100 \mathrm{~nm}$ [80]. Gul-Uludağ et al. loaded specific CD44 siRNA into these polymeric NPs in a 8:1 polymer:siRNA weight ratio [78]. They investigated the silencing efficiency of CD44 in a AML cell line model known to express a high level of CD44 (KG-1 cells). After 3 days, CD44 silencing was significantly superior when CD44 siRNA-loaded polymeric NPs were applied compared with scramble siRNA-loaded polymeric NPs. The cytotoxic activity of CD44 siRNA-loaded polymeric Nps was also more important than the cytotoxicity of the polymer-SCR-siRNA complexes. Experiments were only performed in in vitro models, and need to be continued to confirm the potential of this polymeric formulation compared with the toxicity of CD44-neutralizing antibodies previously reported in clinical trials [81].

Micelles Polymeric micelles are largely used in cancer therapies involving lipophilic drugs [82]. Their pharmacokinetic behavior is driven by the hydrophilic structure of their shell [83]. Polymeric micelles were recently approved by different medicines agencies and are good candidates for solubilizing hydrophobic drugs (notably paclitaxel) without the need for a toxic surfactant. Polymeric micelle formulations of paclitaxel already exist and are used in the clinic (e.g., Genexol-PM ${ }^{\circledR}$, Samyang-Biopham; Nanoxel-M ${ }^{\circledR}$, Samyang-Biopham; and Paclical ${ }$, Oasmia Pharmaceutical AB) [84].

Doxorubicin-loaded polymeric micelles have been developed for AML indications [85]. Dextran was used as a copolymer, and folic acid and retinoic acid were then grafted onto the shell of the micelles to target AML cells. The micelles had a mean size of $80-100 \mathrm{~nm}$ and a encapsulation efficiency of at least $90 \%$. The in vitro cytotoxic activity of doxorubicin-loaded polymeric micelles grafted with folic acid was enhanced in KG-1 cells compared with the free drug. The concentration required to provide $50 \%$ cell death was approximately twice as high using doxorubicin alone compared with doxorubicin-loaded polymeric micelles grafted with folic acid. When folic acid was not grafted, no difference between the free drug and drug-loaded micelles was observed, demonstrating the importance of the folic acid ligand.

More advanced assessments are required to consider micelles for clinical trials in AML treatments.

Dendrimers Dendrimers are 3D branched structures with a nano-size range (Figure 1). They are useful for improving the solubility of hydrophobic drugs. Moreover, a range of terminal functional groups exists and, thus, dendrimers are ideal for drug delivery systems and targeting strategies [86]. Drugs can be conjugated or encapsulated into the core of the dendrimer. In cancer therapy, dendrimers have been used to control drug release and specific drug targeting [87].

No dendrimer particles are currently involved in any clinical trial for AML treatment. However, the literature includes several studies assessing their potential to improve treatment.

Dendrimers for cytarabine encapsulation have been developed by Szulc et al. [88]. First, maltose residues were attached at the amino terminal group of poly(propylene imine). Cytarabine triphosphate was then mixed with dendrimer in phosphate buffer saline, and a complex was finally obtained as a result of ionic interactions between the negatively charged cytarabine and the cationic dendrimer [89]. Cytarabine triphosphate-complexed dendrimers did not significantly decrease HL-60 cell viability compared with cytarabine in solution. By contrast, cytarabine triphosphate-complexed dendrimers significantly increased the cytotoxic potency of cytarabine on the 1301 cell line (a $\mathrm{T}$ cell leukemia cell line). According to the authors, this enhancement resulted from an additional entry or toxicity mechanism [88].

There are no dendrimer-bound chemotherapy formulations currently on the market. For AML indications, few in vitro studies are underway, and these need to be developed through in vivo studies. These formulations also need to be scaled up before clinical applications can be considered.

\section{Lipid-based nanoparticles}

There is a range of lipophilic drugs available but they cannot be solubilized in aqueous media in great quantity. Liposomes, as mentioned above, were originally designed for hydrophilic drugs. Lipophilic drugs can be encapsulated into liposomes as previously described and are then located in the phospholipid bilayer or grafted onto the surface. In the latter case, rapid release of the drug can be observed, and the drug loading is limited. Lipid NPs can be used to prevent this burst release and to enhance drug loading in NPs.

Two main kinds of lipid-based NP are currently being developed; solid lipid NPs (SLNs), with a solid lipid matrix, and nanostructured lipid carriers (NLCs), comprising solid lipids and liquid lipids (Figure 1). In the ideal scenario, lipid-based NPs can be prepared using a low energy process, do not require the use of organic solvent, and can be scaled up [90-92]. Thus, numerous lipophilic cytotoxic drugs have been encapsulated in lipid-based NPs, such as paclitaxel, etoposide, docetaxel, SN38, and doxorubicin; some drugs have been modified to be more lipophilic, such as carbonyl-grafted gemcitabine [93-97]. However, there are currently no lipid-based NPs either on the market or undergoing clinical trials.

Etoposide has been found to be active in de novo relapsed AML [98], and can be used in combination with cytarabine in patients who are either older with AML or have refractory AML [99]. Given its poor water solubility, etoposide in its commercial formulation is only available combined with alcohol and surfactants, such as polysorbate 80 or Cremophor EL, which display cell toxicities. Therefore, etoposide lipid-based nanocarrier formulations were developed for AML indications. Khajavinia et al. developed etoposide-loaded lipid nanocarriers conjugated with transferrin [100]. Particles were prepared by an emulsion-solvent diffusion and evaporation method followed by sonication. The lipid phase, containing etoposide, was dissolved in a mixture of acetone and ethanol, and comprised 
cetylpalmitate, octyldodecanol, soy lecithin, and a fatty amine. A solution of poloxamer 188 was mixed and the emulsion was then ultrasonicated. Finally, transferrin was conjugated to lipid nanocarriers. The size of particles ranged from $158 \mathrm{~nm}$ to $247 \mathrm{~nm}$, depending on the fatty amine and the transferrin conjugation method used. When etoposide was loaded into lipid nanocarriers, its cytotoxic activity on K562 cells was systematically enhanced compared with free etoposide.

Given its lipophilic structure, ATRA is a good candidate for lipid-based NPs, and there are numerous examples in the literature of lipid-based NPs for ATRA encapsulation. One example is ATRA-loaded SLNs obtained by an emulsification-ultrasound process [101,102]. Depending on the components and the oil:surfactant ratio of the SLNs, particles ranged in size from $75 \mathrm{~nm}$ to $>200 \mathrm{~nm}$ [101,102]. In vitro cytotoxicity assessments have been carried out on different acute leukemia cell lines (HL-60, Jurkat, and THP-1) [101]. On the HL-60 cell line, ATRA-loaded SLNs significantly decreased cell viability compared with ATRA in solution. On the Jurkat and THP-1 cell lines, ATRA was inactive, even at high doses. When ATRA was loaded in SLNs, a significant, but modest, decrease in cell viability was observed. Several ATRA-loaded NLCs have also been developed to increase drug loading compared with SLNs. Sabzichi et al. used a high-pressure homogenization technique to produce ATRA-loaded NLCs [103]. A mixture of solid and liquid lipids was produced, followed by the addition of a surfactant (poloxamer 407) under high pressure homogenization. A hot oil-in-water emulsion was produced before it was cooled down to ambient temperature, resulting in particles from 65-115 nm. Chinsriwongkul et al. and Ourique et al. developed NLCs for ATRA encapsulation using an homogenization technique [104,105]. Particles of $200 \mathrm{~nm}$ with a narrow PDI and negative zeta potential were obtained, and the cytotoxicity of the ATRA was increased compared with free solution in HL-60 cells. The concentration required to achieve 50\% cell growth inhibition for ATRA-loaded LNCs was half or less (depending on the oil used to produce the NLCs) that required for an ATRA solution. [104]. Ourique et al. developed NLCs of $213 \mathrm{~nm}$ with an increased lipid-core viscosity to retain ATRA and to reduce ATRA leakage from NLCs. ATRA was dispersed in the lipid core, comprising sorbitan monostearate and a caprylic/capric triglyceride mixture, and demonstrated a similar antiproliferative effect on an AML cell line (HL-60) to that of the free drug [105].

As previously discussed, new targets are being investigated, for example, protein phosphatase 2A (PP2A), which is functionally inactivated in numerous cancers and leukemias [106,107]. Pharmacological restoration of PP2A antagonizes cancer development and progression. Fingolimod, a sphingosine analog (also known as FTY720) is one molecule able to activate PP2A [106]. It is a water-soluble drug with a high oral bioavailability and is FDA approved for patients with multiple sclerosis. Fingolimod has also been evaluated for AML treatment and, according to a systemic evaluation of its safety and toxicity, it appears to be a potential treatment option for AML, because of an acceptable benefit:risk ratio and a lack of bone marrow toxicity [108]. Fingolimod has also been encapsulated in SLNs prepared by hot homogenization followed by sheer homogenization and ultrasonication [109]. NPs of $207 \mathrm{~nm}$ were obtained. Cytotoxic activity in AML cells (HL-60), pharmacokinetics following oral administration (in a mice-based model), and antitumor activity of fingolimod-loaded NPs were evaluated and compared with a fingolimod solution. Fingolimod-loaded SLNs induced a higher percentage of apoptosis. Moreover, oral bioavailability was enhanced by a factor of nine (the maximum drug concentration was enhanced by a factor of 40), and significantly decreased tumor burden in a HL-60 xenografted mouse-based model.

Therefore, NPs could be developed to increase the oral bioavailability of drugs, allowing oral administration of drugs only available by intravenous infusion because of their physicochemical structures. Such strategies have been proposed for hypomethylating agents, such as AZA or DEC, which are only available by subcutaneous or intravenous infusion, respectively. Oral formulations might be more convenient for patients, eliminate injection site reactions, and allow new schedules for improving demethylation [110]. Oral AZA (CC-486) has been assessed in a Phase 1 trial [111] for AML and MDS, and a Phase 3 trial in low-risk MDS is ongoing (NCT01566695), showing the interest in the possible oral administration of such agents. DEC encapsulation in lipid-based NPs for oral administration is currently under investigation [112-114]. For example, after DEC solubilization in Transcutol® HP and Precirol ${ }^{\circledR}$ ATO5, followed by homogenization under high pressure (500-1000 bar) in cold conditions, particles of $116 \mathrm{~nm}$ were obtained by Neupane et al. [113]. An ex vivo permeation study (on an ileum segment) was performed and a fourfold increase in DEC in vitro permeability was observed compared with a DEC solution [112]. When DEC was loaded into an NLC formulation of $30 \mathrm{~nm}$ obtained using a low-energy phase inversion process, its cytotoxicity on two AML cell lines (HEL and HL-60) was enhanced by a factor of 1.5 on HEL cells and 1.8 on HL-60 cells compared with free DEC [114]. Moreover, the apparent permeability of an NCL formulation across a Caco-2 cells monolayer was enhanced by a factor of over nine. The major issue for the clinical development of oral nucleotide analogs is their rapid clearance in the gut and liver by cytidine deaminase. A dose escalation trial of concomitant oral administration of DEC and a cytidine deaminase inhibitor (E7727) has been conducted, with promising results [115].

Lipid-based NPs are currently undergoing in vitro or in vivo assessments with regard to AML treatment. Promising strategies have been suggested to enhance drug targeting, to modify pharmacokinetics of drugs already used in AML treatment, or to modify the administration route to facilitate drug administration or to improve patients' quality of life. New therapeutic strategies, such as fingolimod used for AML indications, are also being developed. As a result of strategies developed to enhance drug loading, and after quality control improvements in the field of lipid NPs, such strategies can be tested in clinical trials. 
Metallic NPs have mostly been developed as part of theranostic strategies, because of a combination of superparamagnetic properties and reactive oxygen species production capabilities used to destroy cancer cells. They can be used as drug carriers because of a covalent conjugation with drugs or imaging agents. Chemotherapeutic drugs generally attached to iron NPs are characterized by low molecular weights, rapid biodistribution, and rapid clearance. Once attached to iron NPs, blood circulation time is increased as well as EPR effects, enhancing tumor retention $[116,117]$. Metallic NPs include iron, gold, silver, zinc, and titanium, and can be produced on a large scale, although they have to demonstrate their good tolerance and the lack of body accumulation over time if chronic administration is to be realized.

No metallic NP formulation is currently under clinical investigation for AML indications. A strategy designed to conjugate cytarabine with iron NPs coated with silica has been presented [118]. Mean particle size varied from 23 $\mathrm{nm}$ to $57 \mathrm{~nm}$ depending on the method used to measure it (microscopy or dynamic light scattering). The cytotoxic capacity of these negatively charged particles $(-11,5 \mathrm{mV})$ was evaluated in AML cell lines (HL-60 and KG-1). An improvement of the antiproliferative effect of cytarabine was observed when it was conjugated with iron NPs. The concentration of NPs required to achieve 50\% cell growth inhibition was half of that required for free cytarabine.

Iron NPs are preferred for their superparamagnetic properties and imaging capability. Using such NPs to conjugate chemotherapies could prove a promising approach for combining treatment and diagnostics. However, there is currently little work assessing this strategy for AML indications.

\section{Concluding remarks}

Here, we have discussed current treatments and innovative formulations (Table 1) that have either been recently approved or are in different stages of development (clinical trials, in vivo or in vitro evaluations) for use against AML. These new formulations are designed to overcome drug resistance or to bypass adverse effects of APIs currently used in chemotherapy (Figure 1). As is the case in other pathologies where NPs are developing, liposomes remain the most advanced area, with ongoing clinical trials and a recent FDA-approved formulation (Vyxeos ${ }^{\circledR}$ ). The second NP category for which future trial investigations are planned is polymeric NPs. Clinical transfer from laboratory investigations using other NP strategies must address inadequate drug loading, toxicity evaluations, and scaling-up issues. As previously reported, the transfer from bench to bedside is a major challenge for nanomedicines [119,120].

Finally, four main research approaches are developing in AML formulation research, namely AML cell line targeting, overcoming drug resistance, modifying the pharmacokinetics of drugs, and modifying administration routes for clinical reasons. If pathology outcomes cannot be modified in the near term for refractory AML, NPs could help to improve patients' quality of life by minimizing pain, inconvenience caused by the administration route, or the adverse effects of treatment. Over the longer term, there is a requirement to ensure the best possible AML outcomes, and combining innovative formulations and new targets recently discovered could contribute to significant improvements. Innovative formulations open the field of personalized therapy in combination with newly identified biomarkers.

Acknowledgments

The authors are very grateful to the Ligue contre le Cancer, and particularly to its Maine et Loire Committee, for its financial support.

Conflicting interest statement

S.T. that he is a consultant to Astellas and Celgene.

References

1 Rodriguez-Abreu, D. et al. (2007) Epidemiology of hematological malignancies. Ann. Oncol. 18 (Suppl.) 1, i3-i8

2 Shah, A. et al. (2013) Survival and cure of acute myeloid leukaemia in England, 1971-2006: a population-based study. Br. J. Haematol. 162, $509-516$

3 Segal, B.H. et al. (2007) Prevention and early treatment of invasive fungal infection in patients with cancer and neutropenia and in stem cell transplant recipients in the era of newer broad-spectrum antifungal agents and diagnostic adjuncts. Clin. Infect. Dis. 44, 402-409

4 McCarthy, M.W. and Walsh, T.J. (2017) Prophylactic measures during induction for acute myeloid leukemia. Curr. Oncol. Rep. 19, 18

5 Sperr, W.R. et al. (2017) Intensive consolidation with G-CSF support: tolerability, safety, reduced hospitalization, and efficacy in acute myeloid leukemia patients >/=60 years. Am. J. Hematol. 92 , e567-e574

6 Sant, M. et al. (2014) Survival for haematological malignancies in Europe between 1997 and 2008 by region and age: results of EUROCARE5, a population-based study. Lancet Oncol. 15, 931-942

7 Pulte, D. et al. (2008) Improvements in survival of adults diagnosed with acute myeloblastic leukemia in the early 21 st century. Haematologica 93, 594-600

8 Sekeres, M.A. et al. (2009) Time from diagnosis to treatment initiation predicts survival in younger, but not older, acute myeloid leukemia patients. Blood 113, 28-36

9 Bertoli, S. et al. (2013) Time from diagnosis to intensive chemotherapy initiation does not adversely impact the outcome of patients with acute myeloid leukemia. Blood 121, 2618-2626

10 Dombret, H. et al. (2015) International phase 3 study of azacitidine vs conventional care regimens in older patients with newly diagnosed AML with $>30 \%$ blasts. Blood $126,291-299$

11 Yates, J.W. et al. (1973) Cytosine arabinoside (NSC-63878) and daunorubicin (NSC-83142) therapy in acute nonlymphocytic leukemia. Cancer Chemother. Rep. 57, 485-488

12 Luger, S.M. (2017) How can one optimize induction therapy in AML? Best Pract. Res. Clin. Haematol. 30, 301-305

13 Lowenberg, B. et al. (2009) High-dose daunorubicin in older patients with acute myeloid leukemia. N. Engl. J Med. 361, 1235-1248

14 Gardin, C. et al. (2013) Superior long-term outcome with idarubicin compared with high-dose daunorubicin in patients with acute myeloid leukemia age 50 years and older. J. Clin. Oncol. 31, 321-327

15 Dohner, H. et al. (2010) Diagnosis and management of acute myeloid leukemia in adults: recommendations from an international expert panel, on behalf of the European LeukemiaNet. Blood 115, 453-474

16 Dohner, H. et al. (2015) Acute myeloid leukemia. N. Engl. J. Med. 373, 1136-52

17 Coombs, C.C. et al. (2015) Acute promyelocytic leukemia: where did we start, where are we now, and the future. Blood Cancer J. 5, e304 
18 Sanz, M.A. et al. (2009) Management of acute promyelocytic leukemia: recommendations from an expert panel on behalf of the European LeukemiaNet. Blood 113, 1875-1891

19 Lo-Coco, F. et al. (2013) Retinoic acid and arsenic trioxide for acute promyelocytic leukemia. N. Engl. J. Med. 369, 111-121

20 Burnett, A.K. et al. (2015) Arsenic trioxide and all-trans retinoic acid treatment for acute promyelocytic leukaemia in all risk groups (AML17): results of a randomised, controlled, phase 3 trial. Lancet Oncol. 16, 1295-1305

21 Platzbecker, U. et al. (2017) Improved outcomes with retinoic acid and arsenic trioxide compared with retinoic acid and chemotherapy in nonhigh-risk acute promyelocytic leukemia: final results of the randomized Italian-German APL0406 Trial. J. Clin. Oncol. 35, 605-612

22 Nassereddine, S. et al. (2017) The role of mutant IDH1 and IDH2 inhibitors in the treatment of acute myeloid leukemia. Ann. Hematol. 96, $1983-1991$

23 Stone, R.M. et al. (2018) Midostaurin: its odyssey from discovery to approval for treating acute myeloid leukemia and advanced systemic mastocytosis. Blood Adv. 2, 444

24 Stone, R.M. et al. (2017) Midostaurin plus chemotherapy for acute myeloid leukemia with a FLT3 mutation. N. Engl. J. Med. 377, 454-464

25 Stein, E.M. et al. (2017) Enasidenib in mutant IDH2 relapsed or refractory acute myeloid leukemia. Blood 130, 722-731

26 DiNardo, C.D. et al. (2016) Determination of IDH1 mutational burden and clearance via next-generation sequencing in patients with IDH1 mutation-positive hematologic malignancies receiving AG-120, a first-in-class inhibitor of mutant IDH1. Blood 128, 1070

27 Konopleva, M. et al. (2016) Efficacy and biological correlates of response in a phase II study of venetoclax monotherapy in patients with acute myelogenous leukemia. Cancer Discov. 6, 1106-1117

28 DiNardo, C.D. et al. (2018) Safety and efficacy of venetoclax (Ven) in combination with decitabine or azacitidine in treatment-naive, elderly patients ( $\geq 65$ years) with acute myeloid leukemia (AML): a non-randomised, open-label, phase 1b study. Lancet Oncol. 19, 216-228

29 Wei, A. et al. (2017) Updated safety and clinical results of phase 1/2 study of Venetoclax plus low-dose cytarabine in treatment-naive acute myeloid leukemia patients aged $\geq 65$ years and unfit for standard induction therapy. In Haematologica

30 Wei, A.H. and Tiong, I.S. (2017) Midostaurin, enasidenib, CPX-351, gemtuzumab ozogamicin, and venetoclax bring new hope to AML. Journal 130, 2469-2474

31 Stein, E.M. and Tallman, M.S. (2016) Emerging therapeutic drugs for AML. Blood 127, 71-78

32 Petersdorf, S.H. et al. (2013) A phase 3 study of gemtuzumab ozogamicin during induction and postconsolidation therapy in younger patients with acute myeloid leukemia. Blood 121, 4854-4860

33 Castaigne, S. et al. (2012) Effect of gemtuzumab ozogamicin on survival of adult patients with de-novo acute myeloid leukaemia (ALFA-0701): a randomised, open-label, phase 3 study. Lancet $379,1508-16$

34 Pemmaraju, N. et al. (2016) Results from phase 2 trial ongoing expansion stage of SL-401 in patients with blastic plasmacytoid dendritic cell neoplasm (BPDCN). Blood 128, 342

35 Fenaux, P. et al. (2009) Efficacy of azacitidine compared with that of conventional care regimens in the treatment of higher-risk myelodysplastic syndromes: a randomised, open-label, phase III study. Lancet Oncol. 10, 223-232

36 Kantarjian, H. et al. (2007) Results of a randomized study of 3 schedules of low-dose decitabine in higher-risk myelodysplastic syndrome and chronic myelomonocytic leukemia. Blood 109, 52-57

37 Kantarjian, H.M. et al. (2012) Multicenter, randomized, open-label, phase III trial of decitabine versus patient choice, with physician advice, of either supportive care or low-dose cytarabine for the treatment of older patients with newly diagnosed acute myeloid leukemia. J. Clin. Oncol. 30, 2670-2677

38 Nieto, M. et al. (2016) The European Medicines Agency Review of decitabine (Dacogen) for the treatment of adult patients with acute myeloid leukemia: summary of the Scientific Assessment of the Committee for Medicinal Products for Human Use. Oncologist 21, 692-700

39 Arber, D.A. et al. (2016) The 2016 revision to the World Health Organization classification of myeloid neoplasms and acute leukemia. Blood 127, 2391-2405

40 Montalban-Bravo, G. and Garcia-Manero, G. (2015) Novel drugs for older patients with acute myeloid leukemia. Leukemia 29, 760-769

41 Saygin, C. and Carraway, H.E. (2017) Emerging therapies for acute myeloid leukemia. J. Hematol. Oncol. 10, 93

42 Stansfield, L.C. and Pollyea, D.A. (2017) Midostaurin: a new oral agent targeting FMS-like tyrosine kinase 3-mutant acute myeloid leukemia. Pharmacotherapy 37, 1586-1599

43 Burnett, A. et al. (2011) Therapeutic advances in acute myeloid leukemia. J. Clin. Oncol. 29, 487-494

44 Mrozek, K. et al. (2012) Prognostic significance of the European LeukemiaNet standardized system for reporting cytogenetic and molecular alterations in adults with acute myeloid leukemia. J. Clin. Oncol. 30, 4515-4523

45 Arrigoni, E. et al. (2016) ATP-binding cassette transmembrane transporters and their epigenetic control in cancer: an overview. Expert Opin. Drug Metab. Toxicol. 12, 1419-1432

46 Raaijmakers, M.H. (2007) ATP-binding-cassette transporters in hematopoietic stem cells and their utility as therapeutical targets in acute and chronic myeloid leukemia. Leukemia 21, 2094-2102

47 Shi, J. et al. (2017) Cancer nanomedicine: progress, challenges and opportunities. Nat. Rev. Cancer 17, 20-37

48 Guo, J. et al. (2017) Antibody-targeted cyclodextrin-based nanoparticles for siRNA delivery in the treatment of acute myeloid leukemia: physicochemical characteristics, in vitro mechanistic studies, and ex vivo patient derived therapeutic efficacy. Mol. Pharm. 14, 940-952

49 Dorrance, A.M. et al. (2015) Targeting leukemia stem cells in vivo with antagomiR-126 nanoparticles in acute myeloid leukemia. Leukemia $29,2143-53$

50 Danhier, F. (2016) To exploit the tumor microenvironment: Since the EPR effect fails in the clinic, what is the future of nanomedicine? J. Control. Release 244, 108-121

51 Tran, S. et al. (2017) Cancer nanomedicine: a review of recent success in drug delivery. Clin. Transl. Med. 6, 44

52 Desai, P.P. et al. (2012) Overcoming poor oral bioavailability using nanoparticle formulations - opportunities and limitations. Drug Discov. Today Technol. 9, e87-e95

53 Morotti, M. et al. (2011) Pharmacokinetic and toxicity considerations for the use of anthracyclines in ovarian cancer treatment. Expert Opin. Drug Metab. Toxicol. 7, 707-720

54 Riganti, C. et al. (2011) Liposome-encapsulated doxorubicin reverses drug resistance by inhibiting P-glycoprotein in human cancer cells. Mol Pharm. 8, 683-700

55 Dicko, A. et al. (2010) Biophysical characterization of a liposomal formulation of cytarabine and daunorubicin. Int. J. Pharm. 391, 248-259

56 Lancet, J.E. et al. (2014) Phase 2 trial of CPX-351, a fixed 5:1 molar ratio of cytarabine/daunorubicin, vs cytarabine/daunorubicin in older adults with untreated AML. Blood 123, 3239-3246

57 Kim, H.P. et al. (2011) Liposomal encapsulation of a synergistic molar ratio of cytarabine and daunorubicin enhances selective toxicity for acute myeloid leukemia progenitors as compared to analogous normal hematopoietic cells. Exp. Hematol. 39, 741-750

58 Cortes, J.E. et al. (2015) Phase II, multicenter, randomized trial of CPX-351 (cytarabine:daunorubicin) liposome injection versus intensive salvage therapy in adults with first relapse AML. Cancer 121, 234-242

59 Brunetti, C. et al. CPX-351 in acute myeloid leukemia: can a new formulation maximize the efficacy of old compounds? Journal 2017, 1-10

60 Lancet, J.E. et al. (2016) Final results of a phase III randomized trial of CPX-351 versus $7+3$ in older patients with newly diagnosed high risk (secondary) AML. J. Clin. Oncol. 34 (Suppl.), 7000 
61 Mayer, L.D. et al. (2006) Ratiometric dosing of anticancer drug combinations: controlling drug ratios after systemic administration regulates therapeutic activity in tumor-bearing mice. Mol. Cancer Ther. 5, 1854-1863

62 Tan, K.B. et al. (2014) Liposomal codelivery of a synergistic combination of bioactive lipids in the treatment of acute myeloid leukemia. Nanomedicine 9, 1665-1679

63 Tan, K.B. et al. (2012) In vivo efficacy of a novel liposomal formulation of safingol in the treatment of acute myeloid leukemia. J. Control. Release 160, 290-298

64 Myhren, L. et al. (2014) Efficacy of multi-functional liposomes containing daunorubicin and emetine for treatment of acute myeloid leukaemia. Eur. J. Pharm. Biopharm. 88, 186-193

65 Moreno, P.M. and Pego, A.P. (2014) Therapeutic antisense oligonucleotides against cancer: hurdling to the clinic. Front. Chem. 2, 87

66 Xin, Y. et al. (2017) Nano-based delivery of RNAi in cancer therapy. Journal 16, 134

67 Adams, B.D. et al. (2017) Targeting noncoding RNAs in disease. J. Clin. Invest. 127, 761-771

68 Tari, A.M. et al. (2007) Liposome-incorporated Grb2 antisense oligodeoxynucleotide increases the survival of mice bearing bcr-abl-positive leukemia xenografts. Int. J. Oncol. 31, 1243-1250

69 Metzeler, K.H. et al. (2013) A stem cell-like gene expression signature associates with inferior outcomes and a distinct microRNA expression profile in adults with primary cytogenetically normal acute myeloid leukemia. Leukemia 27, 2023-2031

70 Pollyea, D.A. and Jordan, C.T. (2017) Therapeutic targeting of acute myeloid leukemia stem cells. Blood 129, 1627-1635

$71 \mathrm{Li}$, H. et al. (2015) CD33-targeted lipid nanoparticles (aCD33LNs) for therapeutic delivery of GTI-2040 to acute myelogenous leukemia. Mol. Pharm. 12, 2010-2018

72 Klisovic, R.B. et al. (2014) Phase I study of GTI-2040, a ribonucleotide reductase antisense, with high dose cytarabine in patients with relapsed/refractory acute myeloid leukemia. Leuk. Lymphoma 55, 1332-1336

73 Bobo, D. et al. (2016) Nanoparticle-based medicines: a review of FDA-approved materials and clinical trials to date. Pharm. Res. 33, 23732387

74 Ashton, S. et al. (2016) Aurora kinase inhibitor nanoparticles target tumors with favorable therapeutic index in vivo. Sci. Transl. Med. 8, $325 \mathrm{ra} 17$

75 Floc'h, N. et al. (2017) Optimizing therapeutic effect of Aurora B inhibition in acute myeloid Leukemia with AZD2811 nanoparticles. Mol. Cancer Ther. 16, 1031-1040

76 Simon, A.M. et al. (2012) Poly (D,L-lactic-co-glycolide) nanoparticles for the improved therapeutic efficacy of all-trans-retinoic acid: a study of acute myeloid leukemia (AML) cell differentiation in vitro. Med. Chem. 8, 805-810

77 Zhu, B. et al. (2017) Novel transferrin modified and doxorubicin loaded Pluronic 85/lipid-polymeric nanoparticles for the treatment of leukemia: In vitro and in vivo therapeutic effect evaluation. Biomed. Pharmacother. 86, 547-554

78 Gul-Uludag, H. et al. (2014) Polymeric nanoparticle-mediated silencing of CD44 receptor in CD34+ acute myeloid leukemia cells. Leuk. Res. $38,1299-1308$

79 Legras, S. et al. (1998) A strong expression of CD44-6v correlates with shorter survival of patients with acute myeloid leukemia. Blood 91, $3401-3413$

80 Landry, B. et al. (2012) Effective non-viral delivery of siRNA to acute myeloid leukemia cells with lipid-substituted polyethylenimines. PLoS ONE 7, e44197

81 Orian-Rousseau, V. (2010) CD44, a therapeutic target for metastasising tumours. Eur. J. Cancer 46, 1271-7

82 Zhang, Y. et al. (2014) Polymeric micelles: nanocarriers for cancer-targeted drug delivery. AAPS Pharm SciTech. 15, 862-871

83 Wang, C. et al. (2013) Pharmacokinetics of polymeric micelles for cancer treatment. Curr. Drug Metab. 14, 900-909

84 Bernabeu, E. et al. (2017) Paclitaxel: What has been done and the challenges remain ahead. Int. J. Pharm. 526, 474-495

85 Varshosaz, J. et al. (2014) Synthesis and characterization of folate-targeted dextran/retinoic acid micelles for doxorubicin delivery in acute leukemia. Biomed. Res. Int. 2014, 525684

86 Wakaskar, R.R. (2017) General overview of lipid-polymer hybrid nanoparticles, dendrimers, micelles, liposomes, spongosomes and cubosomes. J. Drug Target XX, 1-8

87 Abbasi, E. et al. (2014) Dendrimers: synthesis, applications, and properties. Nanoscale Res. Lett. 9, 247

88 Szulc, A. et al. (2016) Sugar-modified poly(propylene imine) dendrimers as drug delivery agents for cytarabine to overcome drug resistance. Int. J. Pharm. 513, 572-583

89 Szulc, A. et al. (2015) Maltose modified poly(propylene imine) dendrimers as potential carriers of nucleoside analog 5'-triphosphates. Int. J. Pharm. 495, 940-947

90 Saliou, B. et al. (2013) Development and in vitro evaluation of a novel lipid nanocapsule formulation of etoposide. Eur. J. Pharm Sci. 50, 172180

91 Heurtault, B. et al. (2002) A novel phase inversion-based process for the preparation of lipid nanocarriers. Pharm. Res. 19, 875-880

92 Battaglia, L. and Gallarate, M. (2012) Lipid nanoparticles: state of the art, new preparation methods and challenges in drug delivery. Expert Opin. Drug Deliv. 9, 497-508

93 Huynh, N.T. et al. (2009) Lipid nanocapsules: a new platform for nanomedicine. Int. J. Pharm. 379, 201-209

94 Moysan, E. et al. (2014) An innovative hydrogel of gemcitabine-loaded lipid nanocapsules: when the drug is a key player of the nanomedicine structure. Soft Matter. 10, 1767-1777

95 Roger, E. et al. (2011) Development and characterization of a novel lipid nanocapsule formulation of Sn38 for oral administration. Eur. J. Pharm. Biopharm. 79, 181-188

96 Antonow, M.B. et al. (2017) Liquid formulation containing doxorubicin-loaded lipid-core nanocapsules: Cytotoxicity in human breast cancer cell line and in vitro uptake mechanism. Mater. Sci. Eng. C Mater. Biol. Appl. 76, 374-382

97 Steiniger, S.C. et al. (2004) Chemotherapy of glioblastoma in rats using doxorubicin-loaded nanoparticles. Int. J. Cancer 109, $759-767$

98 Hartmann, F. et al. (2001) Cytosine arabinoside, idarubicin and divided dose etoposide for the treatment of acute myeloid leukemia in elderly patients. Leuk. Lymphoma 42, 347-355

99 Horikoshi, A. et al. (2008) The value of oral cytarabine ocfosfate and etoposide in the treatment of refractory and elderly AML patients. Int. J. Hematol. 87, 118-125

100 Khajavinia, A. et al. T(2012) argeting etoposide to acute myelogenous leukaemia cells using nanostructured lipid carriers coated with transferrin. Nanotechnology 23, 405101

101 Silva, E.L. et al. (2016) Improved in vitro antileukemic activity of all-trans retinoic acid loaded in cholesteryl butyrate solid lipid nanoparticles. J. Nanosci. Nanotechnol. 16, 1291-1300

102 Castro, G.A. et al. (2009) Formation of ion pairing as an alternative to improve encapsulation and stability and to reduce skin irritation of retinoic acid loaded in solid lipid nanoparticles. Int. J. Pharm. 381, 77-83

103 Sabzichi, M. et al. (2017) Fabrication of all-trans-retinoic acid-loaded biocompatible precirol: a strategy for escaping dose-dependent side effects of doxorubicin. Colloids Surf. B Biointerfaces 159, 620-628

104 Chinsriwongkul, A. et al. (2012) Nanostructured lipid carriers (NLC) for parenteral delivery of an anticancer drug. AAPS PharmSciTech. 13, 150-158 
105 Ourique, A.F. et al. (2010) Lipid-core nanocapsules as a nanomedicine for parenteral administration of tretinoin: development and in vitro antitumor activity on human myeloid leukaemia cells. J. Biomed. Nanotechnol. 6, 214-223

106 Perrotti, D. and Neviani, P. (2013) Protein phosphatase 2A: a target for anticancer therapy. Lancet Oncol. 14, e229-e238

107 Roberts, K.G. et al. (2010) Essential requirement for PP2A inhibition by the oncogenic receptor c-KIT suggests PP2A reactivation as a strategy to treat c-KIT+ cancers. Cancer Res. $70,5438-5447$

108 Enjeti, A.K. et al. (2016) A systematic evaluation of the safety and toxicity of fingolimod for its potential use in the treatment of acute myeloid leukaemia. Anticancer Drugs. 27, 560-568

109 Estella-Hermoso de Mendoza, A. et al. (2015) Lipid nanosystems enhance the bioavailability and the therapeutic efficacy of FTY720 in acute myeloid leukemia. J. Biomed. Nanotechnol. 11, 691-701

110 Mazzaferro, S. et al. (2013) Oral delivery of anticancer drugs I: general considerations. Drug Discov. Today 18, 25-34

111 Garcia-Manero, G. et al. (2011) Phase I study of oral azacitidine in myelodysplastic syndromes, chronic myelomonocytic leukemia, and acute myeloid leukemia. J. Clin. Oncol. 29, 2521-2527

112 Neupane, Y.R. et al. (2013) Lipid drug conjugate nanoparticle as a novel lipid nanocarrier for the oral delivery of decitabine: ex vivo gut permeation studies. Nanotechnology 24, 415102

113 Neupane, Y.R. et al. (2014) Lipid based nanocarrier system for the potential oral delivery of decitabine: formulation design, characterization, ex vivo, and in vivo assessment. Int. J. Pharm. 477, 601-612

114 Briot, T. et al. (2017) Development and in vitro evaluations of new decitabine nanocarriers for the treatment of acute myeloid leukemia. Int J. Nanomedicine 12, 8427-8442

115 Garcia-Manero, G. et al. (2016) Successful emulation of IV decitabine pharmacokinetics with an oral fixed-dose combination of the oral cytidine deaminase inhibitor (CDAi) E7727 with oral decitabine, in subjects with myelodysplastic syndromes (MDS): final data of phase 1 study. Am. Soc. Hematol. XX, YYY-ZZZ

$116 \mathrm{Li}$, K. et al. (2017) Next-generation superparamagnetic iron oxide nanoparticles for cancer theranostics. Drug Discov. Today 22, 1421-1429

117 Sharma, H. et al. (2015) Metal nanoparticles: a theranostic nanotool against cancer. Drug Discov. Today 20, 1143-1151

118 Shahabadi, N. et al. (2016) Improving antiproliferative effect of the anticancer drug cytarabine on human promyelocytic leukemia cells by coating on Fe3O4@SiO2 nanoparticles. Colloids Surf. B Biointerfaces 141, 213-222

119 Venditto, V.J. and Szoka, F.C. Jr (2013) Cancer nanomedicines: so many papers and so few drugs! Adv. Drug Deliv. Rev. 65, 80-88

120 Malhaire, H. and Lagarce, F. (2015) Is the translational approach becoming a reality in nanomedicine? Eur. J. Nanomedicine 7, 79-83 


\section{Author biographies}

\section{Frederic Lagarce}

Frederic Lagarce received his $\mathrm{PhD}$ in 2004, and has been a full professor of pharmaceutical technology and biopharmaceutics at the University of Angers in France since 2012. He is also a hospital pharmacist. His research focuses on translational products (from bench to bedside) and is mainly focused on cancer therapy, especially on bioavailability enhancement by exploiting the interactions between drug products (mainly nanosystems) and living tissues.

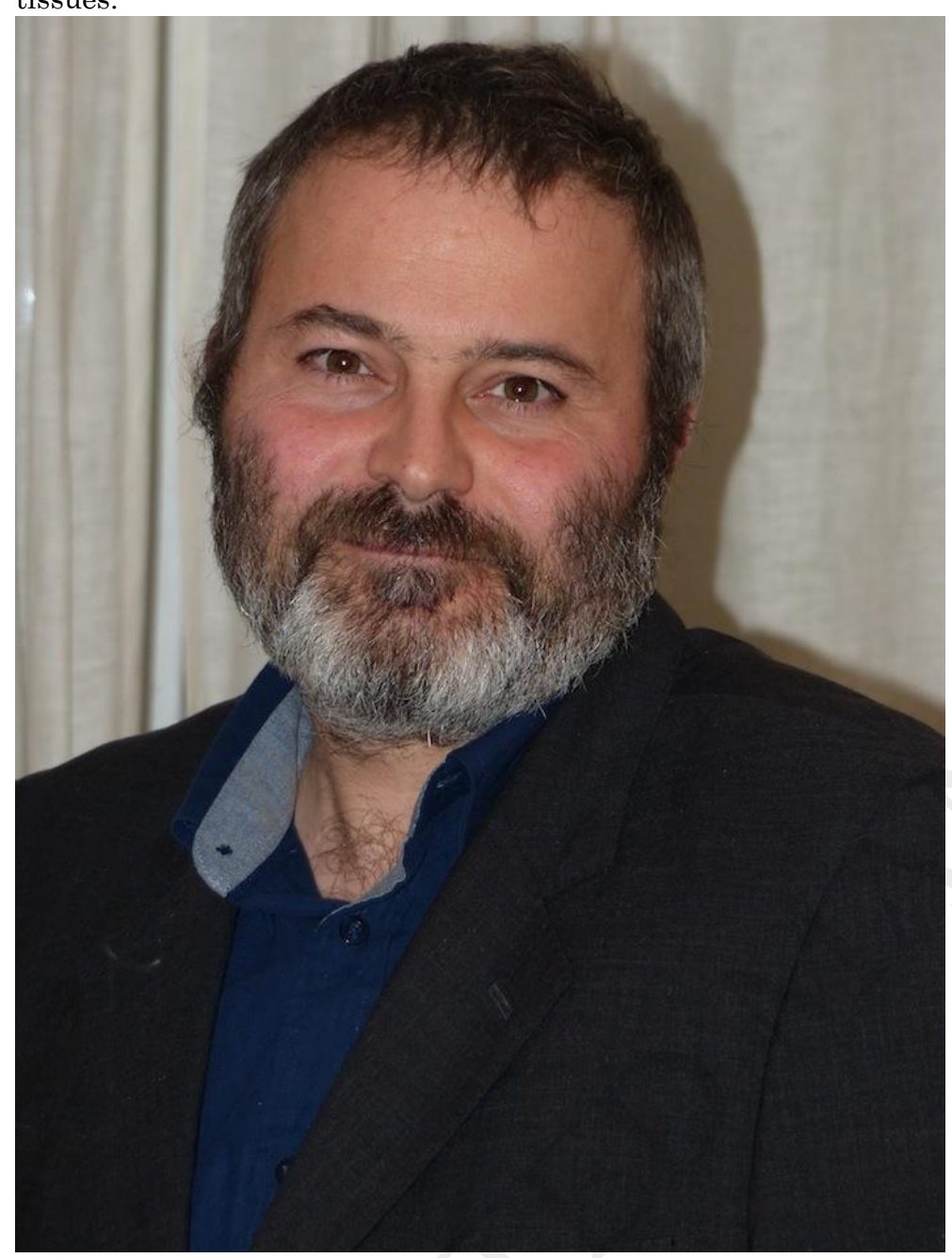

\section{Thomas Briot}

Thomas Briot is a hospital pharmacist and is also currently studying for his $\mathrm{PhD}$ in pharmaceutical technology in the INSERM U1066/CNRS 6021 Unit at the University of Angers. His main interests are drug formulations, analytical quality control, new drug delivery systems (nanomedicines), and oral delivery systems. 


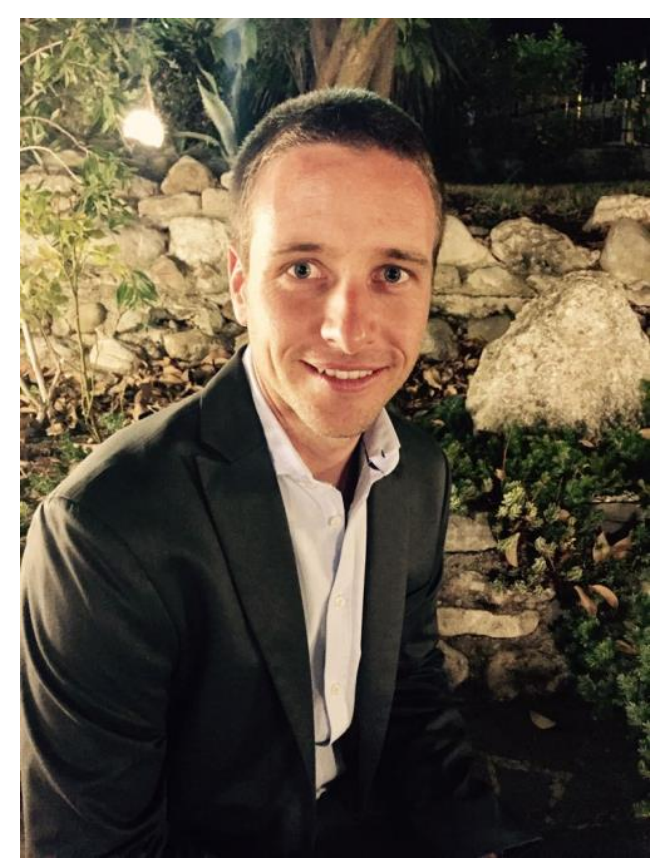




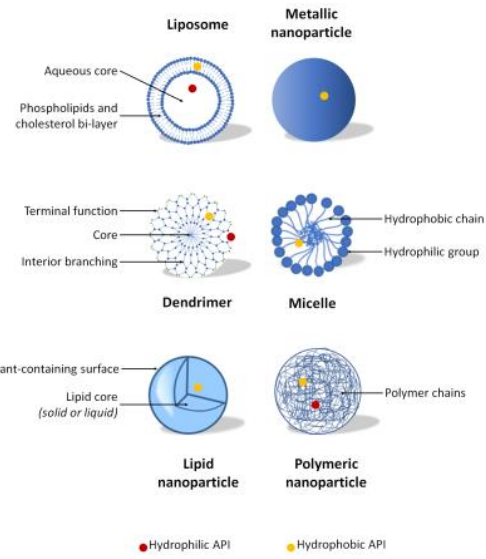

Figure 1. Overview of the six main types of nanocarriers involved in acute myeloid leukemia (AML) therapy at different stages of preclinical or clinical development. Nanocarriers are generally loaded with drugs already used in AML therapy to reduce severe adverse effects and could be functionalized with specific ligands to enhance targeting properties. Abbreviation: API, active pharmaceutical ingredient.

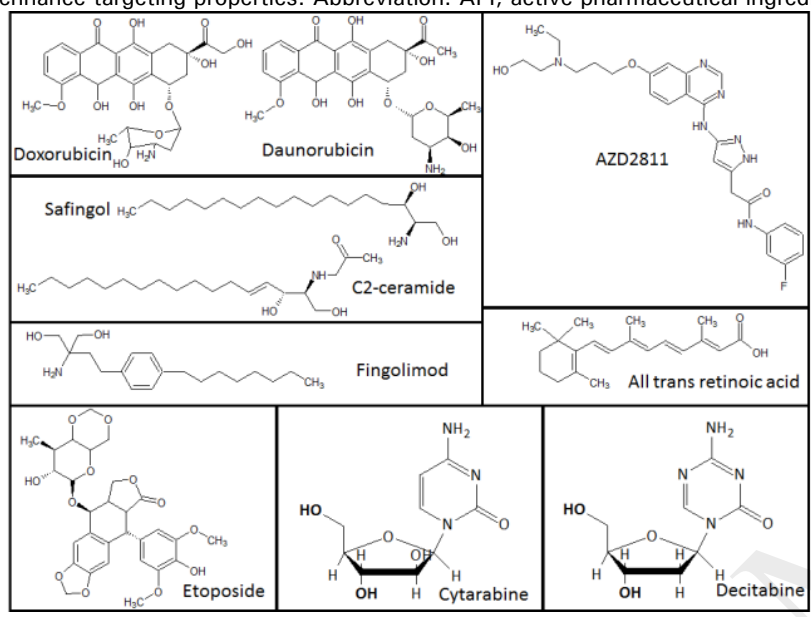

Figure 2. Examples of molecules loaded in innovative formulations developed to treat acute myeloid leukemia (AML) to improve pharmacokinetic parameters as well as reducing adverse effects or modifying the administration route. 
Table 1. Chemotherapeutic drug-loaded nanocarriers involved in clinical trials in December 2017 in an AML indication ${ }^{a}$

Nanocarrier category Drug

Polymeric NPs

Liposomes

AZD2811
Vincristine
Cytarabine-daunorubicin

Cytarabine-daunorubicin

Clinical trial phase Clinical trial ID

1-2

1

2

Not provided

$1-2$

3

Grb2 antisense oligonucleotide 1

Doxorubicin

(1)

Daunorubicin

2

1

$1-2$

3

$1-2$

1-2

2

2-3

3

Cytarabine

NCT03217838

NCT00933985

NCT02337478

NCT01804101

NCT00875693

NCT02642965

NCT02286726/NCT00822094/NCT02019069/NCT00788892/NCT0226957 9/NCT02238925/NCT03335267

NCT01696084

NCT01159028

NCT02781883

NCT00003243

NCT00237627

NCT01736943

NCT00430443

NCT03315039

NCT02722135

NCT00005942

NCT00005962

NCT00111345

NCT02724163/NCT00186966

NCT00003073

NCT00002547

aFrom clinicaltrials.gov, December 2017. 
Table 2. Examples of nanocarriers in current development to improve AML treatment

API class

Anthracyclin

API

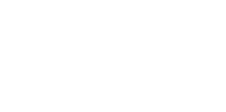

Antipyrimidic Cytarabin

Hypomethylating Decitabine
$\begin{aligned} & \text { agent } \\ & \text { Protein kinase C Safingol } \\ & \text { inhibitors }\end{aligned}$

Oligonucleotide

antisense

C2-ceramide

miR-126

antagonist

GTI-2040

CD44 siRNA

siRNA

based NP

Differentiating

agents

Iron NPs

Lipid NPs

Liposomes based NP

All-trans retinoic NLCs

acid

SLNs

\section{Drug formulation}

Liposomes
Drug association
Yes (cytarabine)

Yes (emetine)

No

Polymeric micelles No

Liposomes Yes

Dendrimers

No

Liposomes

Liposomes

Liposomes

Liposomes

Polymeric NPs

Cyclodextrin-

No

No

No

Yes

ceramide)

Yes (Safingol)

No

No

No

No

No

No
No

\begin{tabular}{llll}
\cline { 3 - 3 } & & & \\
\cline { 3 - 4 } & & Polymeric NPs & No \\
\hline Topoisomerase & II Etoposide & NLCs & No
\end{tabular}

inhibitors

Sphingosine 1- Fingolimod

phosphate receptor

modulators

Aurora B kinase AZD2811

inhibitors
SLNs

No

Polymeric NPs

No
Objectives

Refs

FDA approved and Bypass drug resistance

\section{clinical} trial

investigations

In vitro evaluation

Modify pharmacokinetics; target [64] AML cells

In vivo evaluation Modify pharmacokinetics; target [77]

AML cells; bypass drug resistance

In vitro evaluation Target AML cells [85]

FDA approved Bypass drug resistance

In vitro evaluation Modify pharmacokinetics; bypass $[88,89]$ drug resistance; target AML cells

In vitro evaluation

[118]

Ex vivo, in vitro, and Modify administration route [112-114]

in vitro evaluations (improve patients' quality of life)

Reduce adverse effects; modify [63]

pharmacokinetics

(C2- In vivo evaluation Reduce adverse effects

Teduce adverse effects

$\begin{array}{lll}\text { In vivo evaluation } & \text { Reduce adverse effects } & {[62]} \\ \text { In vivo evaluation } & \text { Target LSCs } & {[49]}\end{array}$

In vivo evaluation Target AML cells

In vitro evaluation Target AML cells; modify $[78,80]$

pharmacokinetics

In vitro and ex vivo Target AML LSCs

evaluation

In vitro evaluation Modify administration route

In vitro evaluation; Modify administration route

$[101,102]$

physicochemical characterization

In vitro evaluation Modify administration route [76]

\begin{tabular}{llll} 
In vitro evaluation & Modify administration route & [76] \\
\hline In vitro evaluation & Target cancerous cells; modify [100]
\end{tabular}

current formulation to reduce

adverse effects

In vivo evaluation Modify pharmacokinetics; target [109]

AML cells

In vivo evaluation Modify pharmacokinetics

$[74,75]$ 